Pontifícia Universidade $_{\text {Do Rio de Janeiro }}$

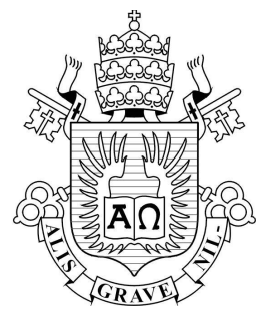

Roberto Machado dos Santos

\title{
Comportamento de Vigas de Concreto Armado Reforçadas com CFC Sujeitas a Carga de Impacto
}

Dissertação de Mestrado

Dissertação apresentada como requisito parcial para obtenção do título de Mestre pelo Programa de PósGraduação em Engenharia Civil da PUC-Rio. Área de Concentração: Estruturas.

Orientador: Giuseppe Barbosa Guimarães

Rio de Janeiro, setembro de 2008 


\title{
Pontifícia Universidade Católica $_{\text {and }}$ DO RIO DE JANEIRO
}

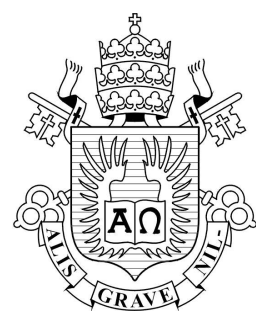

Roberto Machado dos Santos

\section{Comportamento de Vigas de Concreto Armado Reforçadas com CFC sujeitas a Carga de Impacto}

Dissertação apresentada como requisito parcial para obtenção do título de Mestre pelo Programa de PósGraduação em Engenharia Civil da PUC-Rio. Aprovada pela Comissão Examinadora abaixo assinada.

\author{
Giuseppe Barbosa Guimarães \\ Orientador \\ Departamento de Engenharia Civil - PUC-Rio \\ Ibrahim Abd El Malik Shehata \\ Universidade Federal do Rio de Janeiro \\ Paulo Batista Gonçalves \\ Departamento de Engenharia Civil - PUC-Rio
}

José Eugênio Leal

Coordenador Setorial do Centro Técnico Científico - PUC-Rio 
Todos os direitos reservados. É proibida a reprodução total ou parcial do trabalho sem autorização da universidade, do autor e do orientador.

\section{Roberto Machado dos Santos}

Graduou-se em Engenharia Civil na UNIOESTE (Universidade Estadual do Oeste do Paraná). Na UNIOESTE, participou de programas de Iniciação Científica na área de Engenharia Civil. Na PUC-Rio desenvolveu seu trabalho de pesquisa com ênfase em Análise Experimental de Estruturas em Concreto.

Ficha Catalográfica

Santos, Roberto Machado dos

Comportamento de Vigas de Concreto Armado Reforçadas com CFC Sujeitas a Carga de Impacto / Roberto Machado dos Santos; Orientador: Giuseppe Barbosa Guimarães - Rio de Janeiro: PUC, Departamento de Engenharia civil, 2008.

146 f. : il. ; $30 \mathrm{~cm}$

Dissertação (mestrado) - Pontifícia Universidade Católica do Rio de Janeiro, Departamento de Engenharia Civil.

Incluí referências bibliográficas.

1. Engenharia Civil - Teses. 2. Vigas de Concreto. 3. Reforço. 4. Fibras de Carbono. 5. Carga de Impacto. I. Guimarães, Giuseppe Barbosa. II. Pontifícia Universidade Católica. Departamento de Engenharia Civil. III. Título. 


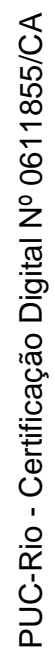

Aos meus pais Junivar e Jucélia, pelo amor e apoio. 


\section{Agradecimentos}

A minha família, pelo carinho ao longo deste trabalho que só foi possível por acreditarem e estarem sempre ao meu lado.

A minha noiva Silmara, pelo apoio e compreensão nos finais de semana dedicados a esta pesquisa, contribuindo ainda na dobra dos estribos.

Ao professor Giuseppe Barbosa Guimarães, pelos ensinamentos prestados e pela orientação recebida ao longo da realização deste trabalho.

Ao meu pai, pelo companheirismo, incentivo, e dedicação fundamental para a realização deste trabalho.

Ao professor Fábio Luiz Willrich (UNIOESTE) pela amizade e por me oferecer condições de percorrer esse caminho.

Ao funcionário Euclídes, pela atenção prestada, muitas vezes por e-mail ou por telefone, na configuração do sistema de Aquisição de Dados.

Ao meu vizinho Hélio Ribeiro, pelo apoio prestado, e dedicação na fabricação do guincho para suspender o martelo, fundamental para a realização dos ensaios.

Ao eng. Gizzi, pela receptividade e suporte disponibilizado para a realização dos ensaios.

A todos os amigos conquistados durante esse período na PUC, por terem sido grandes companheiros durante esta jornada.

Ao CNPQ pelo apoio financeiro.

A todas as pessoas que contribuíram de maneira direta ou indireta para a realização deste trabalho. 


\section{Resumo}

Santos, Roberto Machado dos.; Guimarães, Giuseppe Barbosa (orientador). Comportamento de Vigas de Concreto Armado Reforçadas com CFC Sujeitas a Carga de Impacto. Rio de Janeiro, 2008. 146p. Dissertação de Mestrado - Departamento de Engenharia Civil, Pontifícia Universidade Católica do Rio de Janeiro.

Este trabalho tem como objetivo o estudo experimental do comportamento de vigas de concreto armado reforçadas a flexão com CFC sujeitas a carga de impacto. As variáveis adotadas foram a taxa de carregamento e a taxa de reforço. Todas as vigas foram dimensionadas para resistirem ao mesmo carregamento, de forma que a ruptura fosse governada pelo escoamento do aço da armadura longitudinal de tração. O programa experimental consistiu no ensaio de dezoito vigas biapoiadas de concreto armado. Todas as vigas foram construídas com a mesma seção transversal, vão e resistência de concreto, diferindo somente na armadura longitudinal de tração. Foram confeccionados três tipos de vigas, sendo que as vigas com maior taxa de aço de armadura longitudinal de tração não receberam reforço, enquanto que as demais foram reforçadas com CFC de forma a suportar a mesma carga última das vigas sem reforço. Foram realizados ensaios estáticos e dinâmicos. Para a aplicação da carga de impacto utilizou-se um martelo, que liberado de diferentes alturas de queda, forneceu diferentes taxa de carregamento e, consequentemente, diferentes taxas de deformação nas vigas ensaiadas. Os resultados mostraram que as vigas com reforço de CFC possuem menor capacidade de desaceleração do movimento de queda do martelo. Verificou-se que quanto maior a altura de queda do martelo maior é a força máxima de reação das vigas, apresentando um crescimento praticamente linear com o aumento da taxa de carregamento.

\section{Palavras-chave}

Vigas de Concreto; Reforço; Fibras de Carbono; Carga de Impacto. 


\section{Abstract}

Santos, Roberto Machado dos.; Guimarães, Giuseppe Barbosa (Advisor). Behavior of Reinforced Concrete Beams Strengthened with CFC Under Impact Loading. Rio de Janeiro, 2008. 146p. MSc. Dissertation Civil Engineering Department, Pontifícia Universidade Católica do Rio de Janeiro.

An experimental study on the behavior of reinforced concrete beams strengthened in flexure with CFC under impact load was carried out in this work. The main objective was to investigate the effects of the loading rate on the strength of the beams. The variables were the loading rate and the ratio of fiber to steel reinforcement cross sections. All beams were design to resist the same load, in a way that the failure should be governed by the yielding of the longitudinal tension steel reinforcement. The experimental program consisted of the test of eighteen simply supported beams. All the beams had the same cross section, span and concrete strength. The only difference was the amount of longitudinal tension steel and fiber reinforcements. Static and dynamic testing had been carried through. A hammer was used for the impact load application, which was released from different heights, giving different loads rates and, consequently, different deformation rates in the tested beams. The results showed that the beams with reinforcement of CFC had less capacity of movement deceleration of hammer fall. It was observed that the higher the height of the hammer the higher was the maximum force of reaction of the beams, showing practically a linear growth with the increase of the loading rate.

\section{Keywords}

Concrete Beams; Reinforcement; Carbon Fibers; Impact Load. 


\section{Sumário}

1 Introdução 21

1.1. Considerações Gerais 21

1.2. Objetivos 22

1.3. Organização do Trabalho 22

2 Materiais Compósitos Utilizados em Reforço Estrutural 24

2.1. Introdução 24

2.2. Reforço à Flexão em Vigas de Concreto Armado 26

2.2.1. Critérios de Dimensionamento 26

2.2.2. Modos de Ruptura 29

2.2.3. Aderência do Sistema de Reforço 33

2.3. Análise da Ductilidade 37

2.3.1. Ductilidade de Estruturas Reforçadas com CFC 38

3 Carregamento Produzido por Impacto 41

3.1. Considerações Gerais 41

3.2. Comportamento do Concreto sob Carregamento de Impacto 43

3.2.1. Concreto com adições de fibras 45

3.3. Estudos sobre carregamento dinâmico relacionados a materiais compósitos 46

3.3.1. Estudo de WHITE et al. (2001) 46

3.3.2. Estudo de ERKI E MEIER (1999) 48

3.3.3. Estudo de TANG e SAADATMANESH (2003) 49

3.3.4. Estudo de TANG e SAADATMANESH (2005) 52

3.3.5. Estudo de JEROME e ROSS (1997) 55

4 Programa Experimental $\quad 57$

4.1. Introdução 57

4.2. Materiais 58

4.2.1. Concreto 58 
4.2.2. Aço 60

4.2.3. Compósito de Fibra de Carbono 62

4.3. Vigas 65

4.3.1. Características Geométricas e Mecânicas 65

4.3.2. Dimensionamento do reforço à flexão 66

4.4. Fôrmas 70

4.5. Concretagem 71

4.6. Aplicação do Reforço 73

4.7. Instrumentação das Vigas $\quad 75$

4.7.1. Aquisição de Dados 78

4.8. Aparato para aplicação do Carregamento de Impacto 78

4.9. Execução dos Ensaios das Vigas 86

5 Apresentação e Análise dos Resultados 89

5.1. Introdução

5.2. Ensaios Estáticos $\quad 89$

5.3. Ensaios Dinâmicos 94

5.3.1. Grupo A 95

5.3.2. Grupo B 100

5.3.3. Grupo C 106

5.3.4. Comparação das Forças de Reação Máximas das Vigas 112

5.4. Efeito da Taxa de Carregamento 113

5.5. Efeito da Taxa de Reforço 119

6 Conclusões e Sugestões para Trabalhos Futuros 122

6.1. Conclusões 122

6.2. Sugestões para Trabalhos Futuros 124

7 Referências Bibliográficas 125

$\begin{array}{ll}\text { Anexo A Caracterização dos Agregados } & 130\end{array}$

$\begin{array}{ll}\text { Anexo B Rotina Computacional } & 134\end{array}$

Anexo C Gráficos Comparativos dos Resultados dos Ensaios 138 


\section{Lista de figuras}

Figura 2.1 - Representação esquemática dos PRF.

Figura 2.2 - Diagrama tensão-deformação de diversos tipos de fibras; adaptada de

MACHADO (2006).

Figura 2.3 - Diagrama esquemático dos parâmetros da seção transversal reforçada.

Figura 2.4 - Delaminação do cobrimento de concreto (BEBER et al, 2000). 30

Figura 2.5 - Descolamento do reforço (WHITE et al., 2001). 30

Figura 2.6 - Mecanismo de transferência de esforços entre concreto e reforço; adaptada de MENEGHETTI (2007).

Figura 2.7 - Mecanismo de ruptura da camada de cobrimento; adaptada de ACI $440.2 \mathrm{R}(2002)$

Figura 2.8 - Mecanismo de ruptura prematura devido à fissuras de cisalhamento; adaptada de MACHADO (2006).

Figura 2.9 - Recomendações executivas para as lâminas de CFC; adaptada de ACI 440.2R (2002).

Figura 2.10 - Comparação das parcelas da energia elástica e energia inelástica para uma estrutura com reforço externo e outra sem reforço; adaptada de MACHADO (2004).

Figura 2.11 - Definição do índice de ductilidade em termos de energia; adaptada de MACHADO (2004).

Figura 2.12 - Determinação da inclinação da linha de fechamento do triângulo que define a área da energia elástica; adaptada de MACHADO (2004). 40

Figura 3.1 - Carregamento de impacto em viga biapoiada. 42

Figura 3.2 - Características dos espécimes ensaiados por WHITE et al. (2001). 47

Figura 3.3 - Características dos espécimes ensaiados por ERKI e MEIER (1999).

Figura 3.4 - Esquema de ensaio utilizado por TANG e SAADATMANESH (2003).

Figura 3.5 - (a) Gráfico força de reação máxima vs altura de queda do martelo; (b) Gráfico força de reação máxima vs número de impactos; obtido por TANG e 
SAADATMANESH (2003).

Figura 3.6 - (a) Gráfico deflexão máxima vs altura de queda do martelo; (b) Gráfico deflexão máxima vs número de impactos; obtido por obtido por TANG e SAADATMANESH (2003).

Figura 4.1 - Diagrama tensão x deformação específica das amostras de aço: a) diâmetro nominal de $5 \mathrm{~mm}$; b) diâmetro nominal de $8 \mathrm{~mm}$; a) diâmetro nominal de $10 \mathrm{~mm}$.

Figura 4.2 - Tecido de fibra de carbono, SikaWrap - $230 \mathrm{C}$.

Figura 4.3 - Sikadur 330: a) Componente A; b) Componente B.

Figura 4.4 - Corpos-de-prova de tecido de fibra de carbono revestidos com resina epóxi.

Figura 4.5 - Seção longitudinal das vigas.

Figura 4.6 - Seções transversais das vigas ensaiadas.

Figura 4.7 - Fluxograma para a determinação da capacidade resistente de vigas e o dimensionamento do reforço à flexão.

Figura 4.8 - Fôrmas preparadas: a) primeira concretagem; b) segunda concretagem.

Figura 4.9 - Concretagem das vigas do Grupo A.

Figura 4.9 - Concretagem das vigas do Grupo A (continuação).

Figura 4.10 - Etapas da aplicação do reforço: a) substrato preparado; b) aplicação da primeira camada de resina epóxi; c) rolagem das bolhas de ar; d) aplicação da segunda camada de resina epóxi;

Figura 4.11 - Disposição das ancoragens transversais das vigas. 75

Figura 4.12 - Extensômetro colado na face superior da viga. 76

Figura 4.13 - Colagem e preparação dos extensômetros nas barras de aço. 76

Figura 4.13 - Colagem e preparação dos extensômetros nas barras de aço (continuação).

Figura 4.14 - Colagem de extensômetro em camada de reforço de CFC. $\quad 77$

Figura 4.15 - Esquema de instrumentação das vigas. 78

$\begin{array}{ll}\text { Figura 4.16 - Sistema de aquisição de dados. } & 78\end{array}$

Figura 4.17 - Esquema geral do aparato desenvolvido para a aplicação da carga.

Figura 4.18 - Esquema do sistema desenvolvido para a aplicação da carga: a) 
vista lateral; b) vista frontal.

Figura 4.19 - Apoio das vigas: Detalhe do encaixe da célula-de-carga.

Figura 4.20 - Apoio das vigas: Detalhe da viga sobre os apoios metálicos rotulados.

Figura 4.21 - Martelo utilizado para aplicar a carga de impacto.

Figura 4.22 - Vista superior do equipamento de aplicação da carga de impacto:

Detalhe do encaixe do martelo.

Figura 4.23 - Equipamento utilizado para operar os movimentos do martelo. 84

Figura 4.24 - Roldanas utilizadas para suspender o martelo. 85

Figura 4.25 - Aparato para aplicação da carga de impacto. 86

$\begin{array}{ll}\text { Figura 4.26 - Esquema de aplicação de carga. } & 87\end{array}$

Figura 4.27 - Viga preparada para ensaio: a) dinâmico; b) estático. 88

Figura 5.1 - Ruptura em ensaio estático das vigas: a) VEA; b) VEB; c) VEC. 90

Figura 5.2 - Ruptura do reforço longitudinal de CFC da viga VEC.

Figura 5.3 - Curvas força de reação vs deformação específica: a) viga VEA;

b) viga $\mathrm{VEB}$; c) viga $\mathrm{VEC}$.

Figura 5.3 - Curvas força de reação vs deformação específica: a) viga VEA;

b) viga VEB; c) viga VEC (continuação).

Figura 5.4 - Ruptura das vigas: a) V050DA; b) V100DA; c) V150DA;

d) V200DA; e) V250DA.

Figura 5.4 - Ruptura das vigas: a) V050DA; b) V100DA; c) V150DA; d) V200DA; e) V250DA (continuação).

Figura 5.5 - Curvas deformação específica vs tempo das vigas: a) V050DA;

b) V100DA; c) V150DA; d) V200DA; e) V250DA.

Figura 5.5 - Curvas deformação específica vs tempo das vigas: a) V050DA;

b) V100DA; c) V150DA; d) V200DA; e) V250DA (continuação).

98

Figura 5.6 - Curvas força de reação vs tempo das vigas: a) V050DA; b) V100DA;

c) V150DA; d) V200DA; e) V250DA.

Figura 5.6 - Curvas força de reação vs tempo das vigas: a) V050DA; b) V100DA;

c) V150DA; d) V200DA; e) V250DA (continuação).

100

Figura 5.7 - Ruptura das vigas: a) V050DB; b) V100DB; c) V150DB;

d) V200DB; e) V250DB.

101

Figura 5.8 - Ruptura das barras de aço da armadura longitudinal de tração da 
viga V150DB;

Figura 5.9 - Curvas deformação específica vs tempo das vigas: a) V050DB;

b) V100DB; c) V150DB; d) V200DB; e) V250DB.

Figura 5.9 - Curvas deformação específica vs tempo das vigas: a) V050DB;

b) V100DB; c) V150DB; d) V200DB; e) V250DB (continuação). 103

Figura 5.10 - Curvas força de reação vs tempo das vigas: a) V050DB;

b) V100DB; c) V150DB; d) V200DB; e) V250DB.

Figura 5.10 - Curvas força de reação vs tempo das vigas: a) V050DB;

b) V100DB; c) V150DB; d) V200DB; e) V250DB (continuação). 105

Figura 5.11 - Ruptura das vigas: a) V050DC; b) V100DC; c) V150DC;

d) V200DC; e) V250DC. 106

Figura 5.12 - Ruptura das barras de aço da armadura longitudinal da $\begin{array}{ll}\text { viga V150DC; } & 107\end{array}$

Figura 5.13 - Fissuras na face superior das vigas: a) V150DC; b)V200DC. 108

Figura 5.14 - Curvas deformação específica vs tempo das vigas: a) V050DC;

b) V100DC; c) V150DC; d) V200DC; e) V250DC.

109

Figura 5.14 - Curvas deformação específica vs tempo das vigas: a) V050DC;

b) V100DC; c) V150DC; d) V200DC; e) V250DC (continuação). 110

Figura 5.15 - Curvas força de reação vs tempo das vigas: a) V050DC;

b) V100DC; c) V150DC; d) V200DC; e) V250DC.

Figura 5.15 - Curvas força de reação vs tempo das vigas: a) V050DC;

b) V100DC; c) V150DC; d) V200DC; e) V250DC (continuação). 112

Figura 5.16 - Curvas força máxima de reação vs altura de queda do martelo. 114

Figura 5.17 - Curvas força máxima de reação vs taxa de carregamento. 114

Figura 5.17 - Curvas força máxima de reação vs taxa de carregamento (continuação).

Figura 5.18 - Curvas força de reação vs tempo das vigas que não atingiram a deflexão máxima: a) grupo A; b) grupo B.

Figura 5.19 - Curvas força de reação vs tempo das vigas que atingiram a deflexão máxima: a) grupo $\mathrm{A}$; b) grupo $\mathrm{B}$; c) grupo $\mathrm{C}$.

Figura 5.20 - Curvas deformação específica do aço vs tempo das vigas que não atingiram a deflexão máxima: a) grupo A; b) grupo B.

Figura 5.21 - Curvas força de reação vs tempo das vigas para altura de queda do 
martelo de: a) $0,50 \mathrm{~m}$; b) $1,00 \mathrm{~m}$; c) $1,50 \mathrm{~m}$; d) $2,00 \mathrm{~m}$; e) $2,50 \mathrm{~m}$.

Figura 5.21 - Curvas força de reação vs tempo das vigas para altura de queda do martelo de: a) $0,50 \mathrm{~m}$; b) 1,00m; c) 1,50m; d) 2,00m; e) 2,50m (continuação).

Figura C.1 - Curvas deformação específica do concreto vs tempo conforme os grupos das vigas.

Figura C.2 - Curvas deformação específica do reforço vs tempo conforme os grupos das vigas.

Figura C.3 - Curvas deformação específica do aço vs tempo conforme os grupos das vigas.

Figura C.4 - Curvas deformação específica do concreto vs tempo das vigas conforme as alturas de queda do martelo.

Figura C.4 - Curvas deformação específica do concreto vs tempo das vigas conforme as alturas de queda do martelo (continuação).

Figura C.5 - Curvas deformação específica do reforço vs tempo das vigas conforme as alturas de queda do martelo.

Figura C.5 - Curvas deformação específica do reforço vs tempo das vigas conforme as alturas de queda do martelo (continuação).

Figura C.6 - Curvas deformação específica do aço vs tempo das vigas conforme as alturas de queda do martelo.

Figura C.6 - Curvas deformação específica do aço vs tempo das vigas conforme as alturas de queda do martelo (continuação). 


\section{Lista de tabelas}

Tabela 3.1 - Propriedades físicas e mecânicas dos compósitos laminados. $\quad 49$

Tabela 4.1 - Identificação e nomenclatura das vigas ensaiadas. 58

Tabela 4.2 - Consumo de materiais por metro cúbico de concreto. 59

Tabela 4.3 - Valores médios de resistência do concreto à compressão. 60

Tabela 4.4 - Dados obtidos nos ensaios à tração das barras de aço. 62

Tabela 4.5 - Dados obtidos nos ensaios à tração dos materiais compósitos de fibra de carbono. $\quad 64$

Tabela 4.6 - Dados teóricos das vigas no Estado Limite Último de Ruptura. $\quad 70$

Tabela 5.1 - Resultados experimentais e teóricos dos ensaios estáticos. 91

Tabela 5.2 - Valores obtidos nos ensaios das vigas do grupo A. 98

Tabela 5.3 - Valores obtidos nos ensaios das vigas do grupo B. 104

Tabela 5.4 - Valores obtidos nos ensaios das vigas do grupo C. 110

Tabela 5.5 - Comparação das forças de reação máximas das vigas. 113

Tabela A.1 - Composição granulométrica - agregado miúdo. 130

Tabela A.2 - Composição granulométrica - agregado graúdo. 131 


\section{Lista de símbolos}

\section{Romanos}

$A_{c} \quad$ Área da seção transversal da viga

$A_{f} \quad$ Área da seção transversal do CFC longitudinal

$A_{s c} \quad$ Área de aço da seção da armadura longitudinal de compressão

$A_{s t} \quad$ Área de aço da seção da armadura longitudinal de tração

$A_{w f} \quad$ Área da seção transversal do CFC aplicado como reforço transversal

$b_{f} \quad$ Largura do compósito de fibra de carbono

$b_{w} \quad$ Largura da seção da viga

c Cobrimento de concreto da armadura

CFC Compósito de fibra de carbono

d Altura útil da seção

$d_{f} \quad$ Distância entre a força resultante da seção comprimida de concreto e o reforço à flexão

$D_{\max } \quad$ Dimensão máxima característica do agregado

$E_{c s} \quad$ Módulo de elasticidade secante do concreto

$E_{e l} \quad$ Energia elástica

$E_{f} \quad$ Módulo de elasticidade do compósito de fibra de carbono

$E_{\text {inel }} \quad$ Energia inelástica

$E_{s} \quad$ Módulo de elasticidade do aço

$E_{\text {tot }} \quad$ Energia total

$f_{c} \quad$ Tensão de ruptura à compressão do concreto

$f_{f} \quad$ Tensão do compósito de fibra de carbono

$f_{f e} \quad$ Tensão efetiva do CFC aplicado como reforço transversal 
$f_{s c} \quad$ Tensão no aço da armadura longitudinal de compressão

$f_{\text {st }} \quad$ Tensão no aço da armadura longitudinal de tração

$f_{y c} \quad$ Tensão de escoamento do aço da armadura longitudinal de compressão

$f_{y t} \quad$ Tensão de escoamento do aço da armadura longitudinal de tração

$F_{I} \quad$ Força de impacto

$g \quad$ Aceleração da gravidade

$h \quad$ Altura da viga

$h_{0} \quad$ Altura de queda

I Momento de inércia

$I_{I I} \quad$ Momento de inércia da viga no Estádio II

$k_{m} \quad$ Fator redutor da deformação específica de ruptura do material compósito de fibra de carbono

$k_{1} \quad$ Coeficiente função da resistência à compressão do concreto

$k_{2} \quad$ Coeficiente função do esquema de reforço transversal adotado

$K_{b s} \quad$ Rigidez linear

$K_{v} \quad$ Coeficiente redutor da deformação específica de ruptura do CFC aplicado como reforço transversal

l Vão da viga

L Comprimento da viga

$L_{e} \quad$ Comprimento de aderência do CFC

LN Distância da linha neutra à borda comprimida do concreto

$m_{I} \quad$ Massa do impactor

$\bar{m} \quad$ Massa por unidade de comprimento 


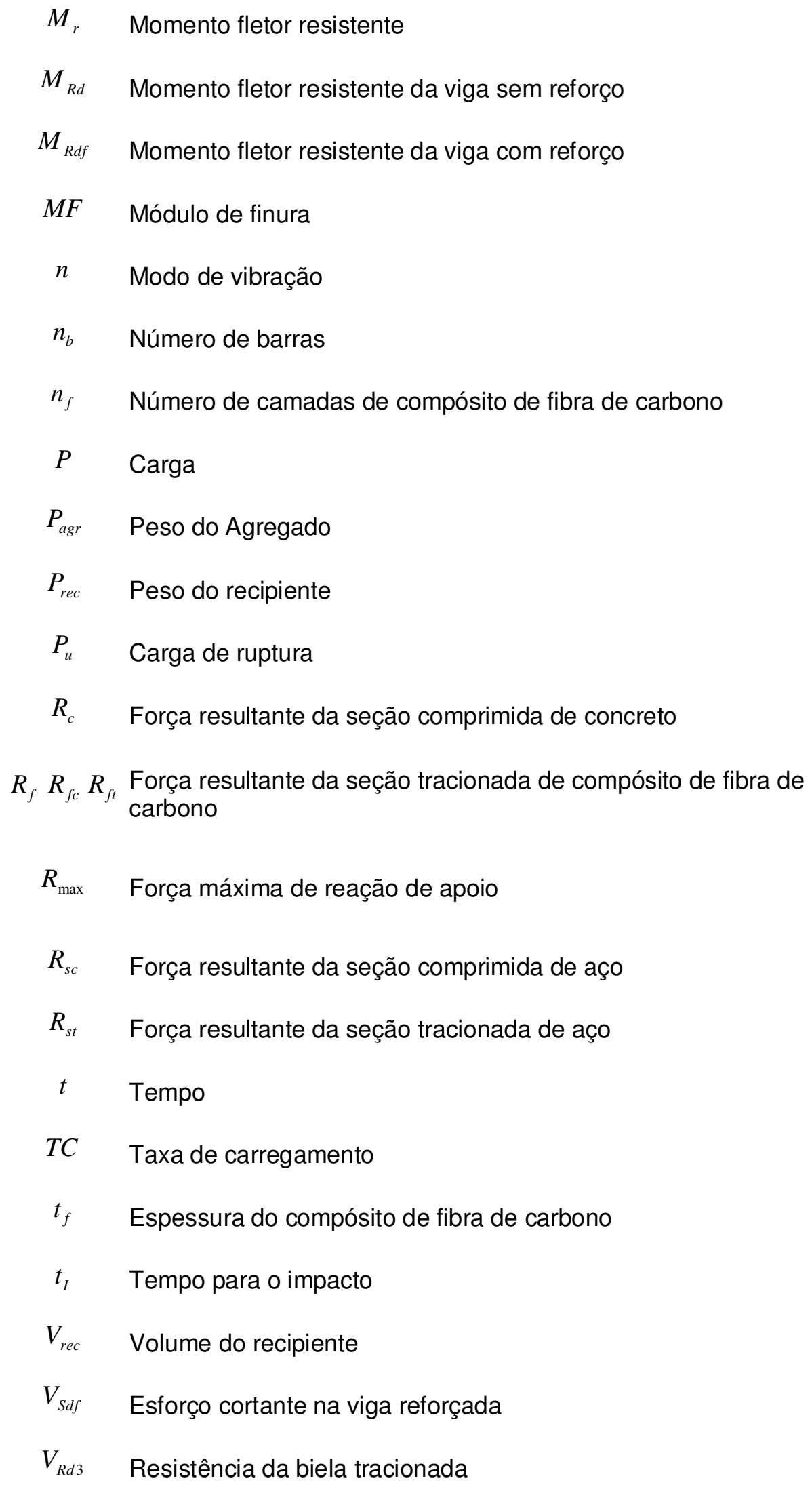


$x \quad$ Posição a partir da extremidade da viga

\section{Gregos}

$\delta \quad$ Flecha da viga

$\delta_{y} \quad$ Flecha da viga para o escoamento do aço da armadura longitudinal de tração

$\delta_{u} \quad$ Flecha da viga para a carga de ruptura

$\dot{\delta} \quad$ Velocidade do impactor no momento do impacto

$\Delta V \quad$ Esforço solicitante no CFC aplicado como reforço transversal

$\varepsilon \quad$ Deformação específica

$\varepsilon_{c} \quad$ Deformação específica do concreto

$\mathcal{E}_{f} \quad$ Deformação específica do compósito de fibra de carbono

$\varepsilon_{f e} \quad$ Deformação específica efetiva do compósito de fibra de carbono aplicado como reforço transversal

$\varepsilon_{f u} \quad$ Deformação específica última do compósito de fibra de carbono

$\varepsilon_{s c}$ Deformação específica do aço da armadura longitudinal de
compressão

$\varepsilon_{s t} \quad$ Deformação específica do aço da armadura longitudinal de tração

$\varepsilon_{s y} \quad$ Deformação específica de escoamento do aço

$\phi \quad$ Diâmetro

$\gamma_{e} \quad$ Massa específica do agregado

$\gamma_{u} \quad$ Massa unitária compactada do agregado

$\eta \quad$ Nível de reforço à flexão

$\varphi \quad$ Função de forma

$\mu \quad$ Índice de ductilidade energética

$v \quad$ Deslocamento da extremidade da viga

$\dot{v} \quad$ Velocidade da extremidade da viga no momento do impacto 
$\rho_{\mathrm{s}} \quad$ Taxa geométrica de aço da armadura longitudinal de tração

$\sigma \quad$ Tensão

$\omega \quad$ Freqüência de vibração

$\psi \quad$ Fator redutor da força resultante da seção comprimida de concreto 